\title{
Impacts of Sodium/Glucose Cotransporter-2 Inhibitors on Circulating Uric Acid Concentrations: A Systematic Review and Meta-Analysis
}

\author{
Abolfazl Akbari, ${ }^{1}$ Mahdi Rafiee, ${ }^{1}$ Thozhukat Sathyapalan, ${ }^{2}$ \\ and Amirhossein Sahebkar ${ }^{3,4,4,5,6}$ \\ ${ }^{1}$ Student Research Committee, Faculty of Medicine, Mashhad University of Medical Sciences, Mashhad, Iran \\ ${ }^{2}$ Department of Academic Diabetes, Endocrinology and Metabolism, Hull York Medical School, University of Hull, Hull, UK \\ ${ }^{3}$ Applied Biomedical Research Center, Mashhad University of Medical Sciences, Mashhad, Iran \\ ${ }^{4}$ Biotechnology Research Center, Pharmaceutical Technology Institute, Mashhad University of Medical Sciences, Mashhad, Iran \\ ${ }^{5}$ Department of Medical Biotechnology and Nanotechnology, Faculty of Medicine, Mashhad University of Medical Sciences, \\ Mashhad, Iran \\ ${ }^{6}$ Department of Biotechnology, School of Pharmacy, Mashhad University of Medical Sciences, Mashhad, Iran
}

Correspondence should be addressed to Amirhossein Sahebkar; amir_saheb2000@yahoo.com

Received 20 October 2021; Accepted 24 January 2022; Published 17 February 2022

Academic Editor: Marco Infante

Copyright (C) 2022 Abolfazl Akbari et al. This is an open access article distributed under the Creative Commons Attribution License, which permits unrestricted use, distribution, and reproduction in any medium, provided the original work is properly cited.

\begin{abstract}
Background. Several trials have assessed the antihyperglycemic effects of sodium/glucose cotransporter-2 inhibitors (SGLT2i) in patients with type 2 diabetes mellitus (T2DM). We conducted a quantitative analysis to assess the impact of SGLT2is on serum uric acid (SUA) in patients with T2DM. Methods. Placebo-controlled trials published before 13 August 2021 were identified by searching PubMed, Embase, Web of Science, and Scopus. The intervention group received SGLT2i as monotherapy or add-on treatment, and the control group received a placebo that was replaced with SGLT2i. Clinical trials providing changes in SUA were included. The mean change of SUA, glycated hemoglobin (HbAlc), fasting plasma glucose (FPG), and body weight were calculated (PROSPERO CRD42021287019). Results. After screening of 1172 papers, 59 papers were included in the systematic review. A total of 55 trials (122 groups) of 7 types of SGLT2i on patients with T2DM were eligible for meta-analysis. All SGLT2is significantly decreased SUA levels compared with the placebo groups: empagliflozin mean difference $(\mathrm{MD})=-40.98$ $\mu \mathrm{mol} / \mathrm{L}, 95 \%$ CI $[-47.63,-34.32]$, dapagliflozin $\mathrm{MD}=-35.17 \mu \mathrm{mol} / \mathrm{L}, 95 \%$ CI $[-39.68,-30.66]$, canagliflozin $\mathrm{MD}=-36.27 \mu \mathrm{mol} /$ $\mathrm{L}, 95 \% \mathrm{CI}[-41.62,-30.93]$, luseogliflozin $\mathrm{MD}=-24.269 \mu \mathrm{mol} / \mathrm{L}, 95 \% \mathrm{CI}[-33.31,-15.22]$, tofogliflozin $\mathrm{MD}=-19.47 \mu \mathrm{mol} / \mathrm{L}$, 95\% CI [-27.40, -11.55], and ipragliflozin MD $=-18.85 \mu \mathrm{mol} / \mathrm{L}, 95 \%$ CI $[-27.20,-10.49]$. SGLT2i also decreased FPG, body weight, and HbA1c levels. SUA reduction persisted during long-term treatment with SGLT2i (except for empagliflozin), while the SUA reduction was affected by the duration of diabetes. Conclusions. SGLT2i can be a valid therapeutic strategy for patients with T2DM and comorbid hyperuricemia. Besides reducing FPG, body weight, and HbA1c, SGLT2i can significantly decrease SUA levels compared to placebo (Total MD $=-34.07 \mu \mathrm{mol} / \mathrm{L}, 95 \%$ CI $[-37.00,-31.14]$ ).
\end{abstract}

\section{Introduction}

Sodium/glucose cotransporter-2 inhibitors (SGLT2i) are one of the main classes of medications that are used for the management of patients with type 2 diabetes (T2DM) [1]. They also have significant renoprotective and cardioprotective properties [2-4]. These oral glucose-lowering agents have been shown to reduce the risk of cardiovascular and renal complications in patients with T2DM $[2,3,5-7]$ plus several other salutary effects on autophagy pathways, neuromodulatory pathways, oxidative stress pathways, platelet function, blood pressure, and hepatic function $[5,8-12]$. 
Dapagliflozin, canagliflozin, ipragliflozin, empagliflozin, sotagliflozin, tofogliflozin, ertugliflozin, and luseogliflozin are some of the established SGLT2is. The action of SGLT2is is independent of insulin; they reduce the renal glucose reabsorption mediated by the SGLT2 expressed along the proximal tubules [6]. Several randomized controlled trials (RCTs) with placebo-controlled groups studied the efficacy of SGLT2is in patients with and without T2DM. The change in serum uric acid (SUA) is one of the parameters which is directly or indirectly assessed in RCTs [13-72]. Increased SUA (hyperuricemia) is an important risk factor for cardiovascular and renal complications of T2DM [73, 74]. Hence, lowering SUA levels with SGLT2is could be a valid therapeutic strategy in this cohort of patients [9-12]. Di Zhao et al. [75] evaluated the effect of empagliflozin on SUA levels through a metaanalysis of clinical trials published before December 2017. They found that empagliflozin reduced serum uric acid levels and other cardiometabolic risk factors such as glycated hemoglobin (HbA1c), fasting plasma glucose (FPG), systolic and diastolic blood pressures, and body weight. Di Zhao and his team did not review other SGLT2is. A meta-analysis by Xin et al. [76] showed that SGLT2is could benefit patients with T2DM with increased SUA levels. However, this manuscript reviewed studies published before August 2017. Several recently published RCTs on the effects of SGLT2is on SUA need to be evaluated in a new meta-analysis. Moreover, limiting RCTs to placebo-controlled ones may help to identify uratelowering properties that can be solely attributed to SGLT2i. The present study was aimed at finding any changes in SUA levels in individuals on SGLT2i based on randomized, placebo-controlled trials.

\section{Materials and Methods}

The current systematic review and meta-analysis were conducted according to the recommendations of the Preferred Reporting Items for Systematic Review and Meta-Analysis Protocols (PRISMA) [77]. This review was registered in PROSPERO (registration number: CRD42021287019).

2.1. Data Sources and Searches. The electronic databases of PubMed, Embase, Scopus, and Web of Science were searched to identify eligible clinical trials using relevant search terms to "Sodium-glucose cotransporter-2 inhibitors (SGLT2i)" and " uric acid" by A.A. and M.R.; complete search strategy is available in Table S1. We identified articles published up to May 5, 2021, without restrictions on language and year of publication. In addition, we updated the article on August 13, 2021. Two authors (A.A. and M.R.) did a further manual search of the references lists of all selected papers, previous similar reviews, and pooled analysis studies to look for possible missing papers.

2.2. Study Selection. The two investigators (A.A. and M.R.) selected the studies according to the following criteria: (1) population: subjects (regardless of their disease) using any kind of SGLT2i; (2) intervention: SGLT2is monotherapy or as an add-on to other antidiabetic medications; (3) compar- ison: SGLT2is were replaced with placebo; (4) outcome: serum uric acid changes; (5) design: clinical trials; and (6) follow-up duration: at least 4 weeks. We excluded from our meta-analysis studies that were not conducted on patients with T2DM. The conference abstracts and pooled analysis studies were carefully assessed for possible duplicate data. Furthermore, several studies assessed serum uric acid at different time points. We chose the time point that was closer to 24 weeks.

2.3. Data Extraction and Quality Assessment. The two investigators (A.A. and M.R.) independently extracted the following data: first author, year of publication, type of study population, number of participants, demographic data, intervention (type of SGLT2i and dose regimen), follow-up duration, duration of diabetes, baseline estimated glomerular filtration rate (eGFR), and outcome (change in SUA, HbAlc, body weight, and FPG from baseline). Moreover, these authors assessed the quality of studies using the quality criteria proposed by the Joanna Briggs Institute (JBI) checklist [40]. If any disagreements existed, these were resolved through discussion or referral to another investigator (A.H.S.). Checklist questions were answered by "yes," "no," "unclear," or "not/applicable." Each "yes" answer takes 1 point. After adding up the scores, the studies were classified into three groups based on their risk of bias: high risk of bias (scores between 0 and 5), intermediate-risk (scores between 6 and 10), and low-risk groups (scores between 11 and 13).

2.4. Publication Bias and Statistical Analysis. Publication bias was examined using funnel plots, Egger's test and Begg's test. Mean differences (MD) and 95\% confidence interval (CI) in SUA levels were calculated using a random-effects model to evaluate the effects of SGLT2is on SUA, HbA1c, body weight, and FPG. Heterogeneity was calculated using $I^{2}$, with $I^{2}$ values $>50 \%$ representing moderate heterogeneity. $P$-value less than 0.05 was considered as statistically significant for the outcome and heterogeneity analyses. Random-effect meta-regression analysis was done to assess the effects of the patient's duration of diabetes, treatment period, and SGLT2i dosage on SUA level changes. Data analysis was done using the Comprehensive Meta-Analysis software (CMA) V.3.

\section{Results}

A total of 1920 papers were collected during the initial electronic search. Through a manual search, six papers were identified. Among those papers, 754 were duplicates, so the 1172 remaining papers were assessed for eligibility criteria. Finally, 59 trials met the inclusion criteria, and 55 trials were included in the meta-analysis. The screening, assessing, and analyzing steps are shown in Figure 1. Seven types of SGLT2is were assessed, including canagliflozin, dapagliflozin, empagliflozin, ipragliflozin, tofogliflozin, ertugliflozin, and luseogliflozin. Descriptive characteristics of the 59 included trials ( 9 types of SGLT2is) are presented in Table 1.

3.1. Outcome. Of the 36,215 patients, 23,494 received different SGLT2is in different dosages versus 12,721 patients who 


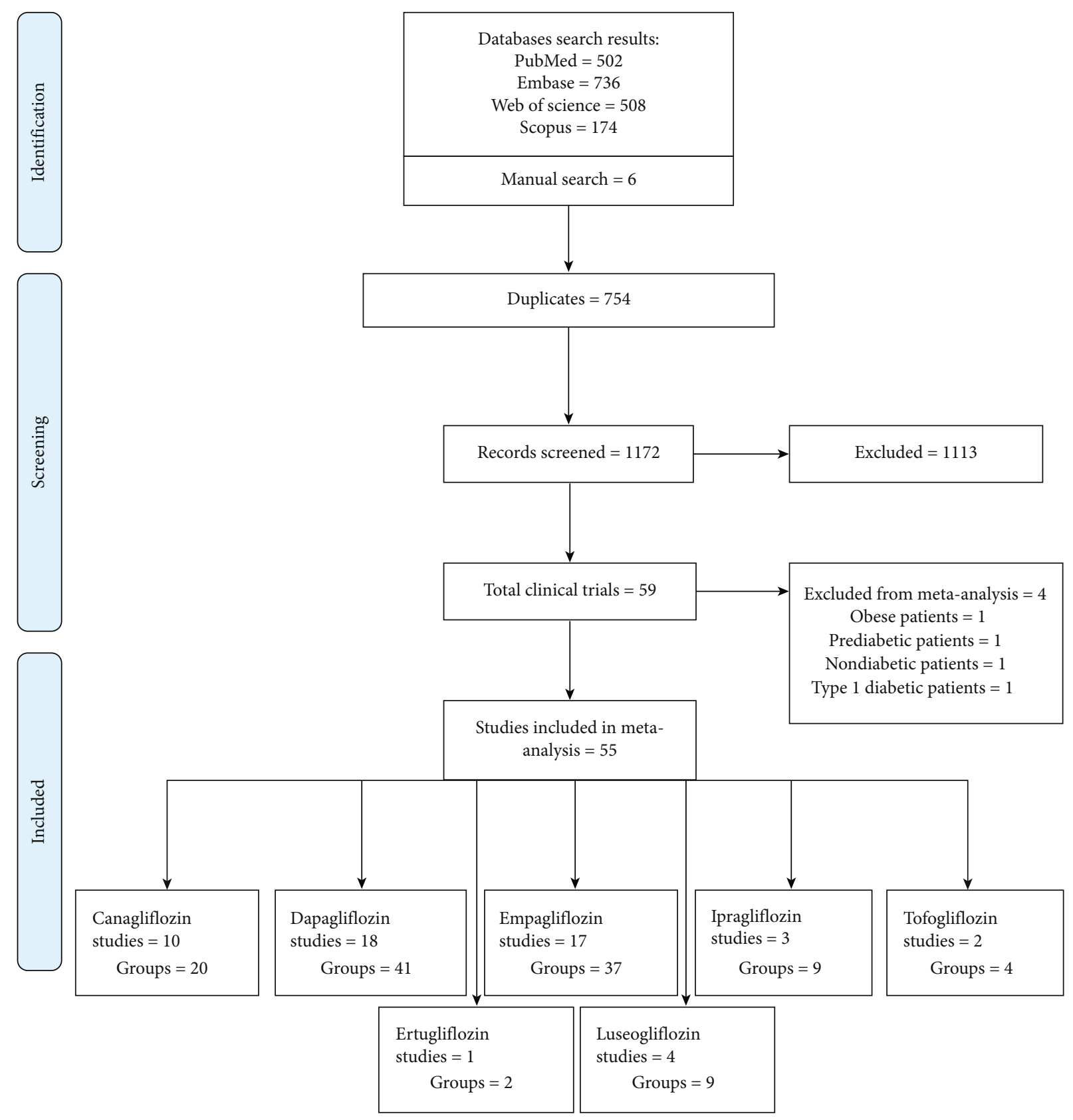

Figure 1: PRISMA flow diagram.

received placebo. The effect size, population, and heterogeneity of SGLT2is included in meta-analysis are shown in Table 2. SGLT2is considerably decreased SUA levels compared with placebo (Total MD, $-34.07 \mu \mathrm{mol} / \mathrm{L}, 95 \%$ CI [-37.00, -31.14], empagliflozin MD, $-40.98 \mu \mathrm{mol} / \mathrm{L}, 95 \%$ CI $[-47.63,-34.32]$, dapagliflozin $\mathrm{MD},-35.17 \mu \mathrm{mol} / \mathrm{L}, 95 \%$ CI $[-39.68,-30.66]$, canagliflozin $\mathrm{MD},-36.27 \mu \mathrm{mol} / \mathrm{L}, 95 \% \mathrm{CI}$ $[-41.62,-30.93]$, luseogliflozin MD, $-24.269 \mu \mathrm{mol} / \mathrm{L}, 95 \%$ CI $[-33.31,-15.22]$, tofogliflozin $\mathrm{MD},-19.47 \mu \mathrm{mol} / \mathrm{L}, 95 \%$ CI $[-27.40,-11.55]$, and ipragliflozin $\mathrm{MD},-18.85 \mu \mathrm{mol} / \mathrm{L}$, 95\% CI $[-27.20,-10.49]$ ) (Figures S1-S3 and Figures 2-7).

Out of 122 comparisons between the different dosages of SGLT2is and placebo, 21 comparisons showed that SGLT2is did not significantly reduce the SUA. After the removal of studies which were conducted only on patients with chronic kidney disease (CKD) $[22,43,68,72]$, the MD of SUA changes of dapagliflozin compared to placebo increased to $-36.29 \mu \mathrm{mol} / \mathrm{L}$ (95\% CI [-40.53, -32.05], $\left.I^{2}=69.3 \%\right)$, the MD of SUA changes of canagliflozin compared to placebo increased to $-37.44 \mu \mathrm{mol} / \mathrm{L}$ (95\% CI [-42.90, -31.97], $I^{2}=$ $68.0 \%$ ), and MD of SUA changes of empagliflozin compared to placebo increased to $-43.79 \mu \mathrm{mol} / \mathrm{L}$ (95\% CI $[-50.75$, $-36.83], I^{2}=85.9 \%$ ).

3.1.1. Canagliflozin. Ten clinical trials evaluated the effect of canagliflozin (range of $50 \mathrm{mg}$ to $600 \mathrm{mg}$ ) on SUA. Canagliflozin $300 \mathrm{mg}$ reduced the SUA, FPG, body weight, and $\mathrm{HbA1c}$ more than canagliflozin $100 \mathrm{mg}$ (Table 2). Moreover, 


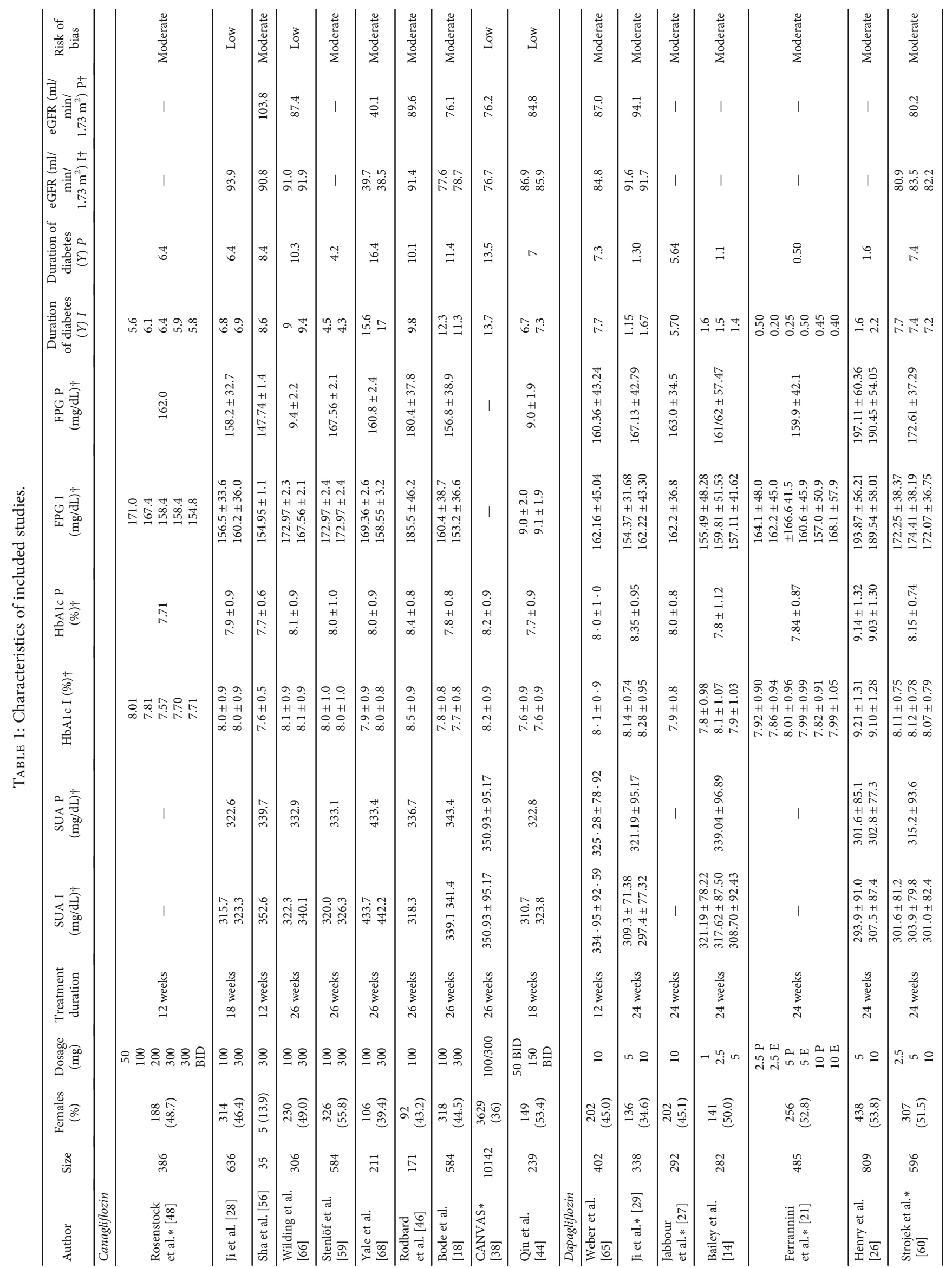




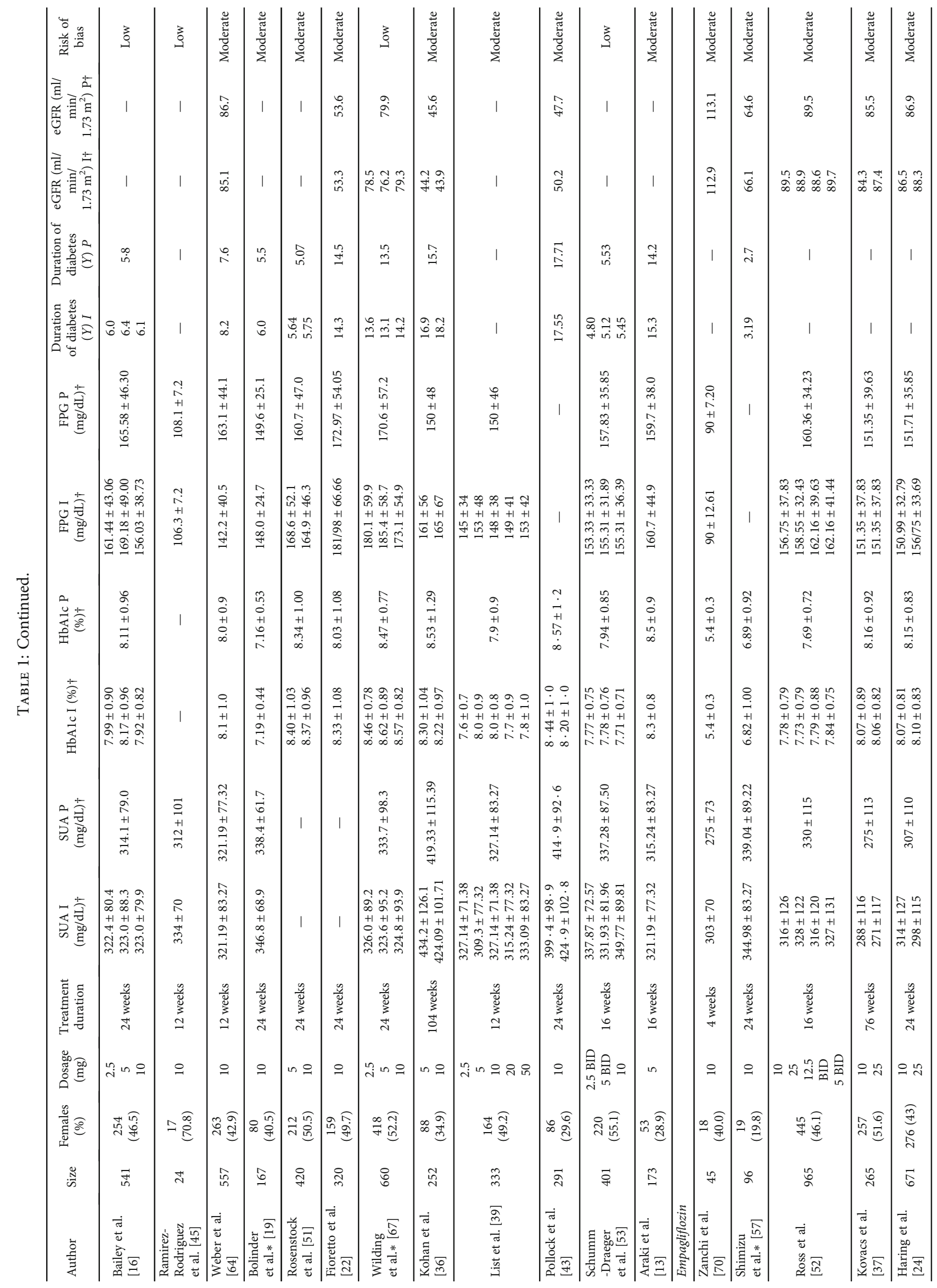




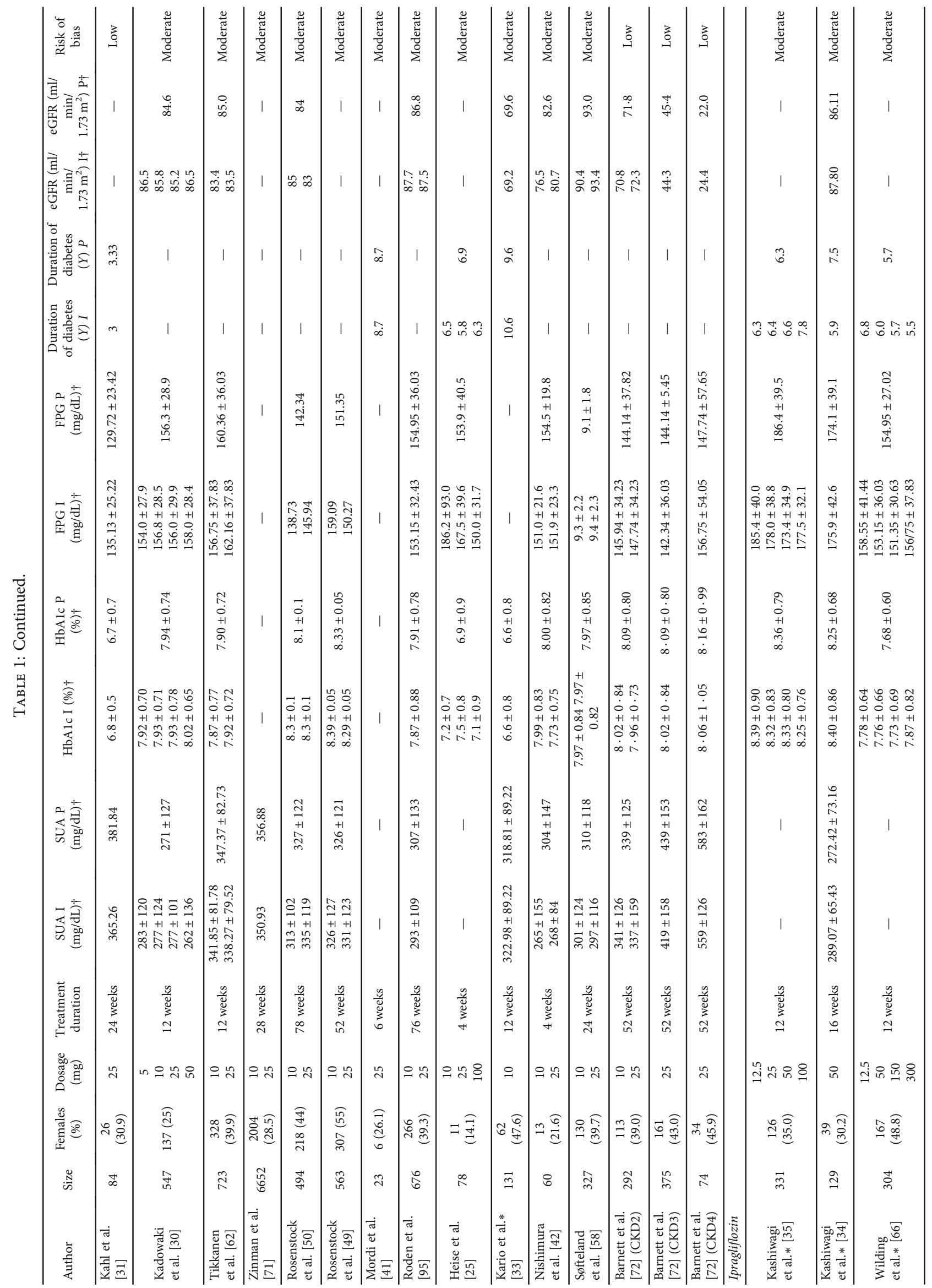




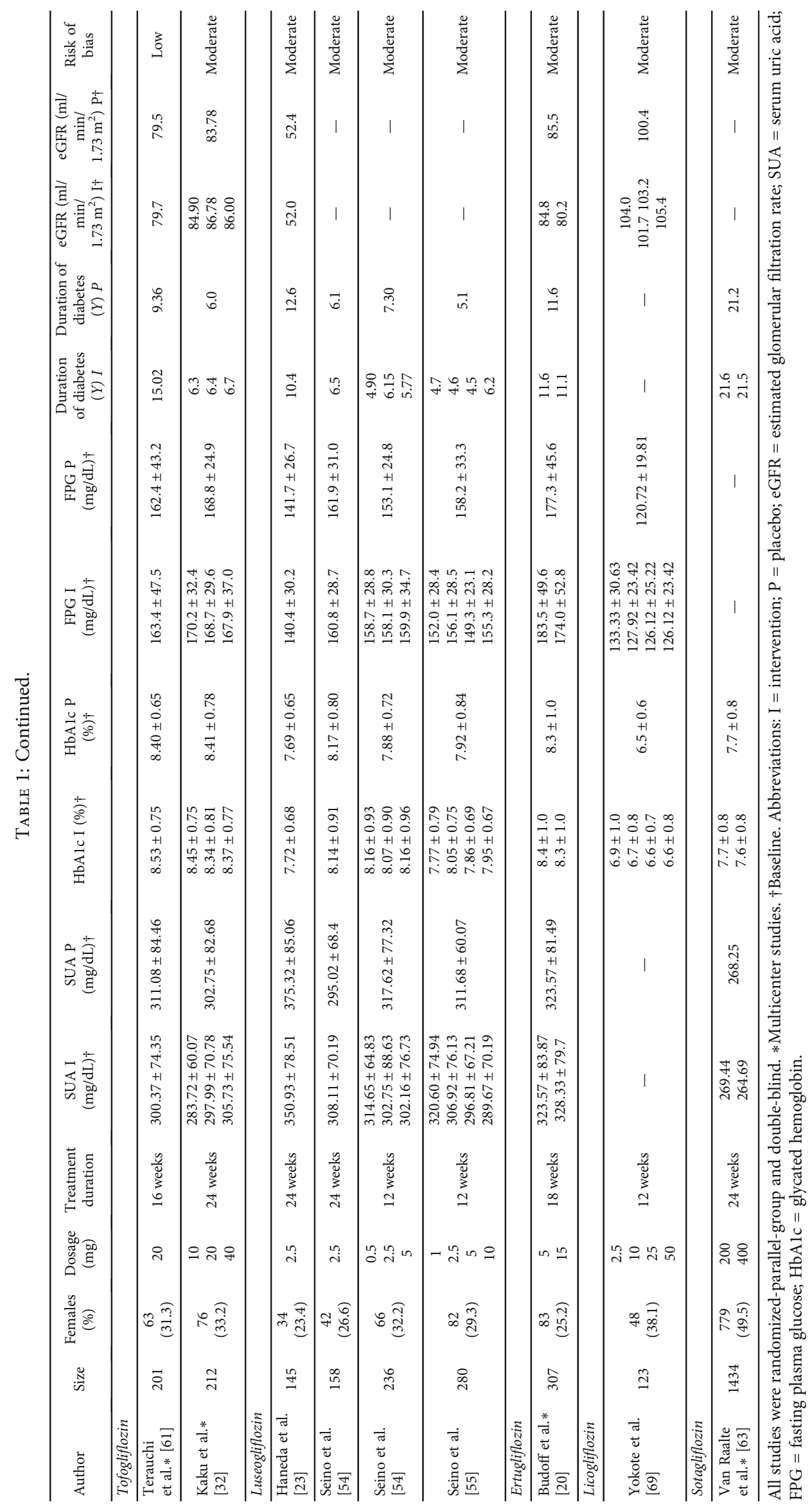




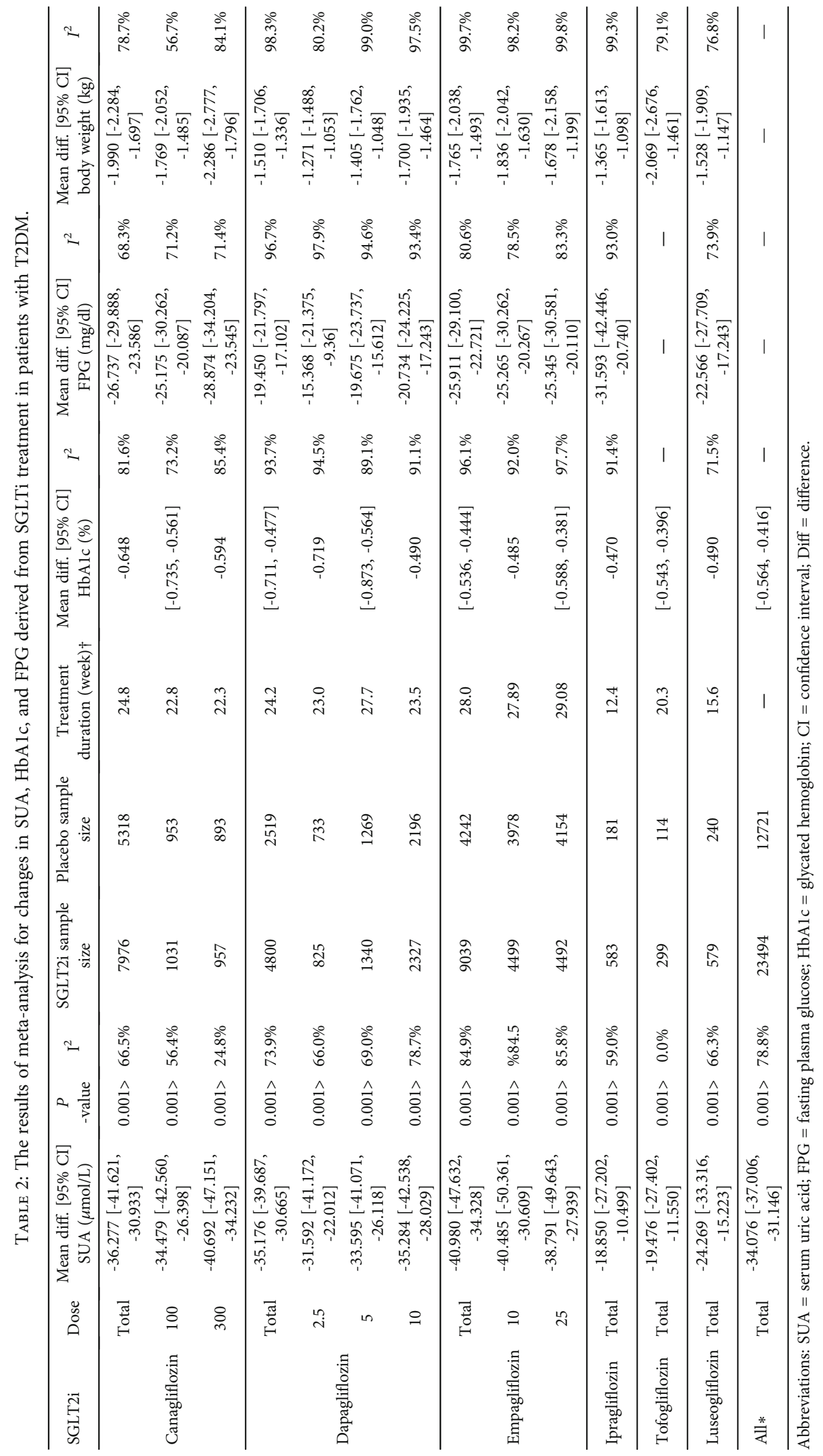



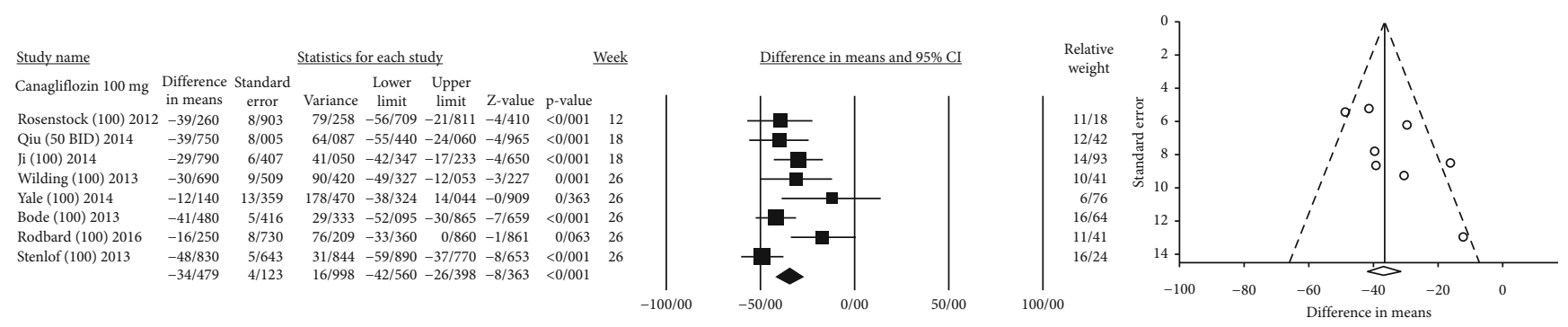

(a)
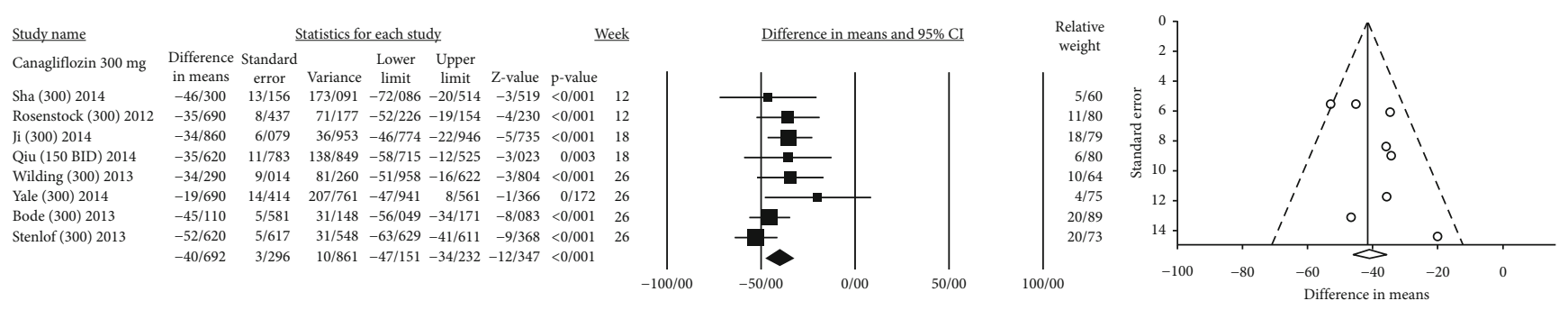

(b)

Figure 2: Mean difference and 95\% confidence intervals for changes in serum uric acid level for canagliflozin compared to placebo ((a) canagliflozin $100 \mathrm{mg}$; and (b) canagliflozin $300 \mathrm{mg}$ ).
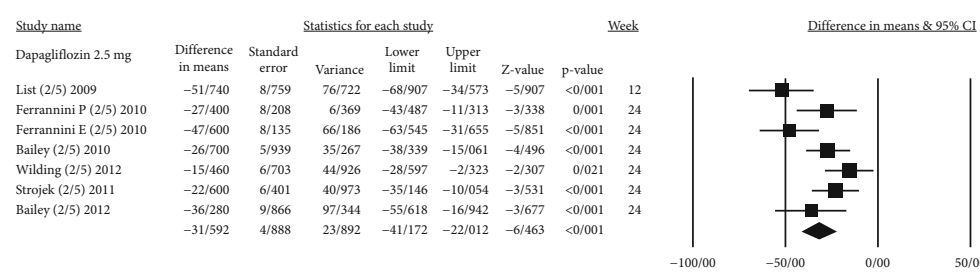

(a)
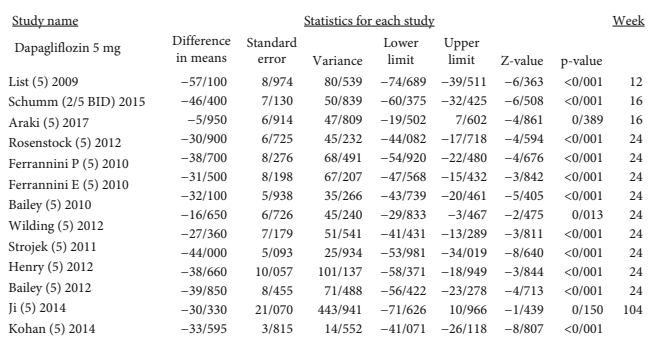

$\underline{\text { Week }}$
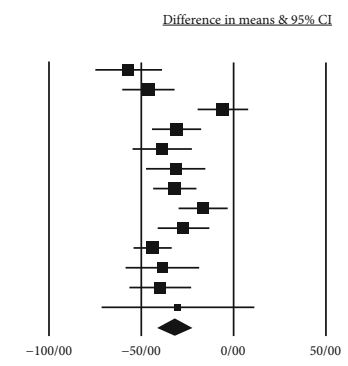

(b)
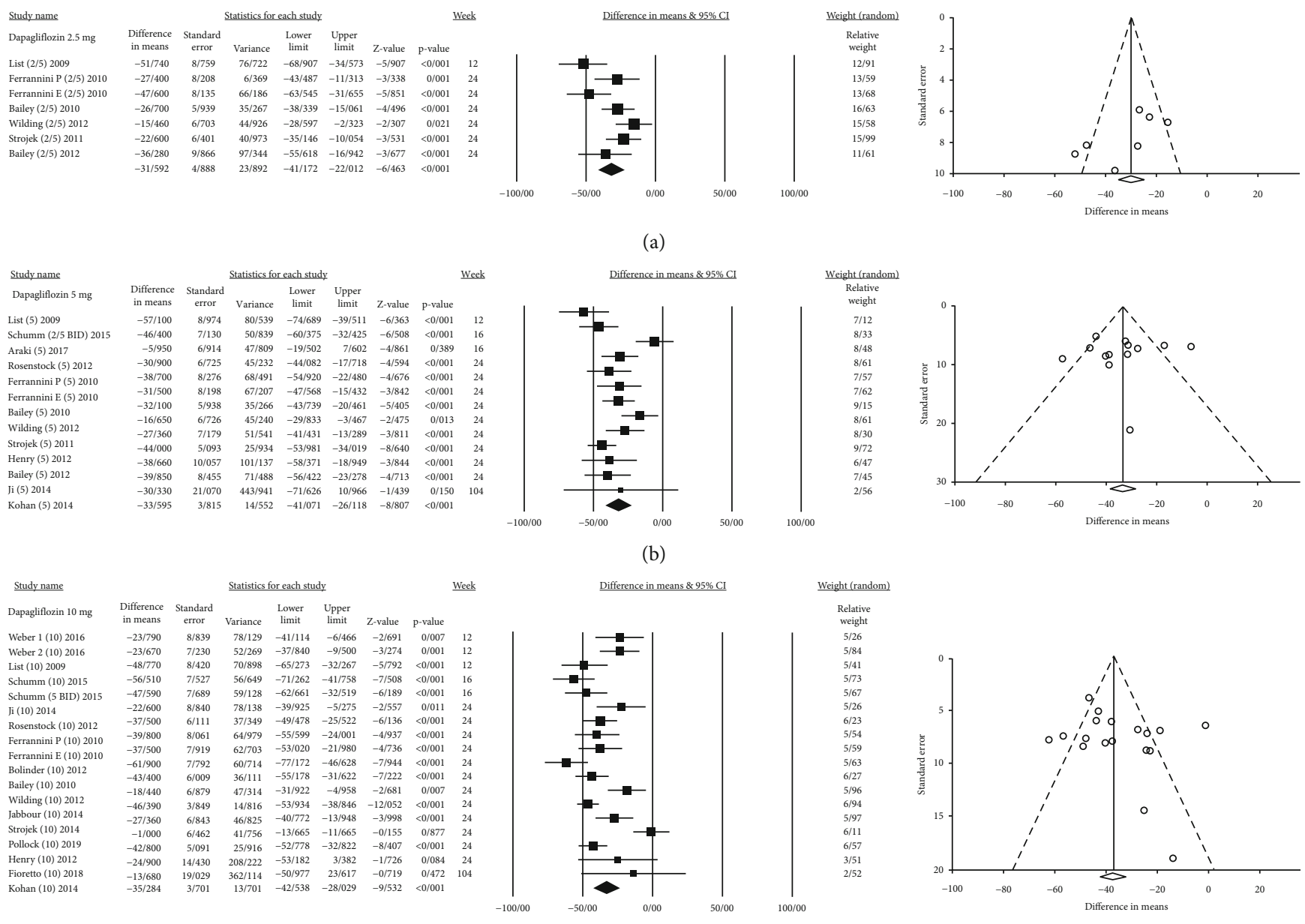

(c)
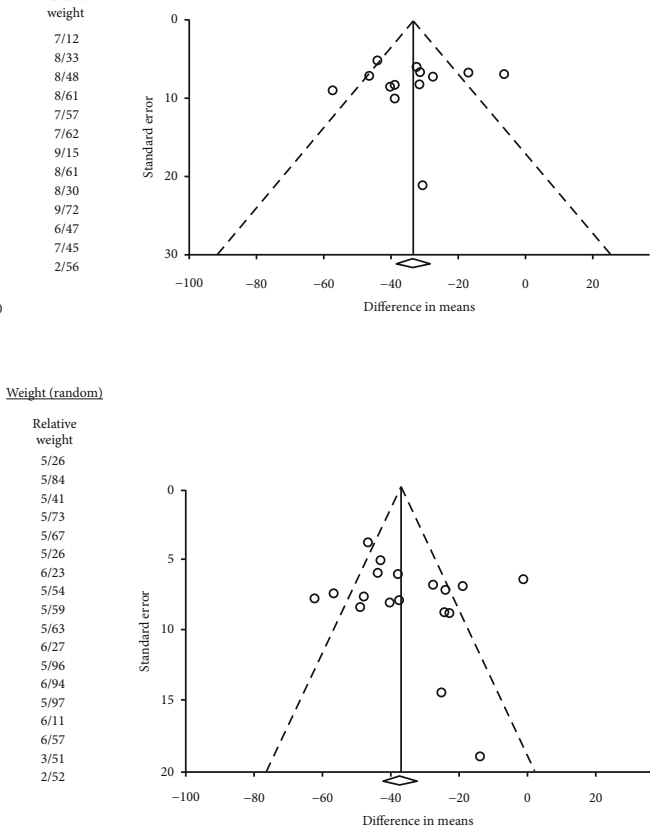

Figure 3: Mean difference and 95\% confidence intervals for changes in serum uric acid level for dapagliflozin compared to placebo ((a) dapagliflozin $2.5 \mathrm{mg}$; (b) dapagliflozin $5 \mathrm{mg}$; and (c) dapagliflozin $10 \mathrm{mg}$ ). 

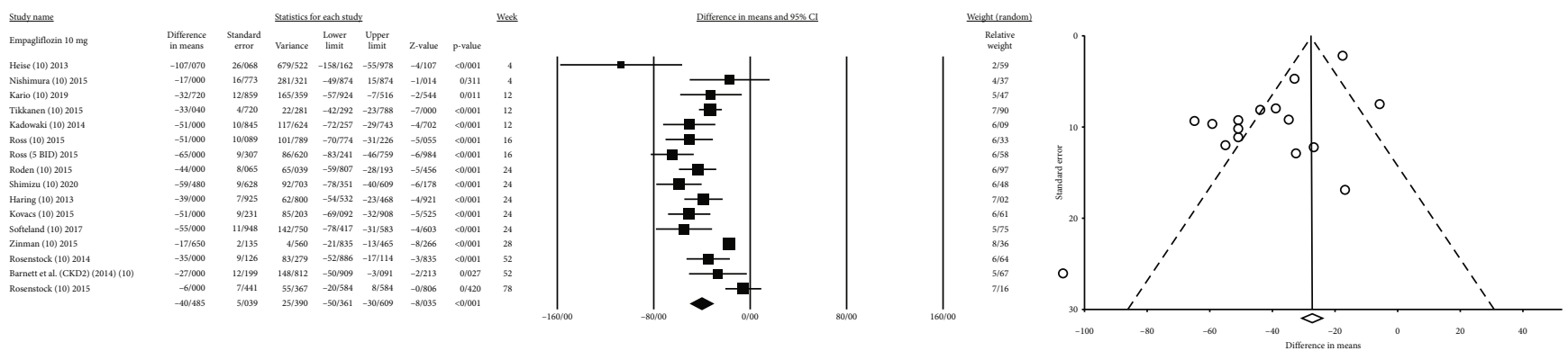

(a)
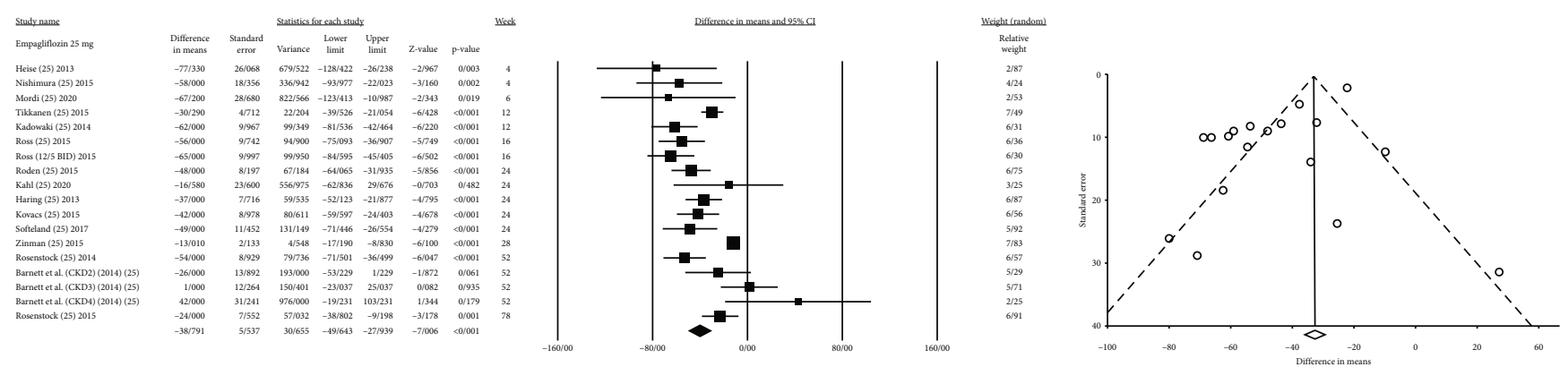

(b)

FIGURE 4: Mean difference and 95\% confidence intervals for changes in serum uric acid level for empagliflozin compared to placebo ((a) empagliflozin $10 \mathrm{mg}$; and (b) empagliflozin $25 \mathrm{mg}$ ).
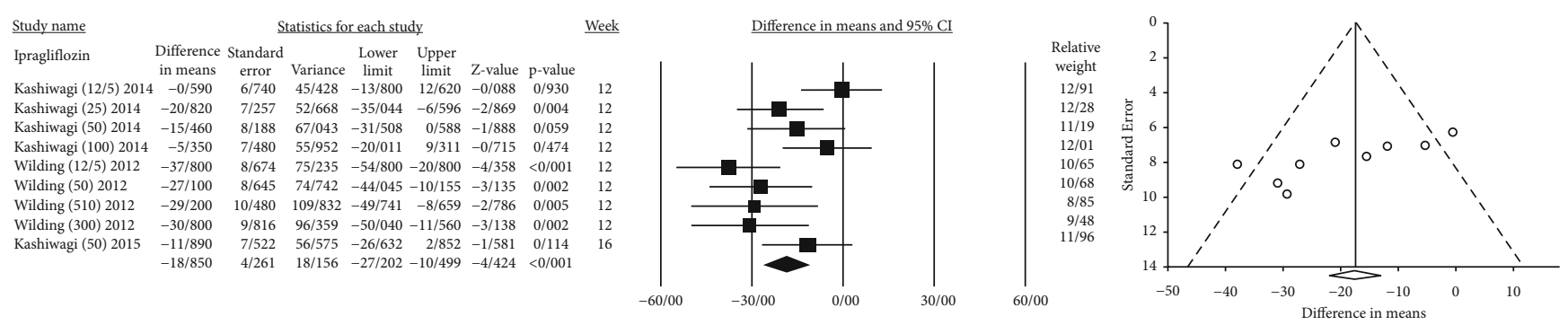

FIGURE 5: Mean difference and 95\% confidence intervals for changes in serum uric acid level for ipragliflozin (range of $12.5 \mathrm{mg}$ to $300 \mathrm{mg}$ ) compared to placebo.
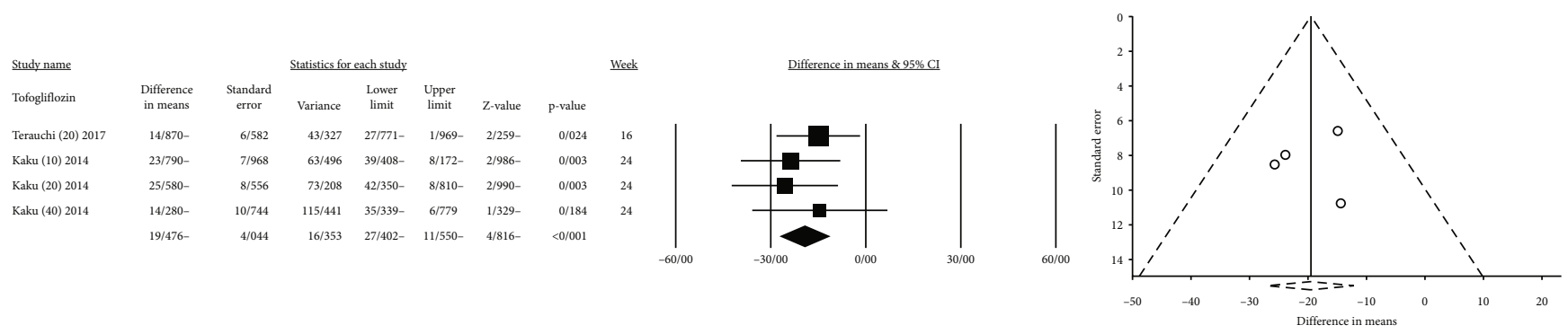

FIGURE 6: Mean difference and 95\% confidence intervals for changes in serum uric acid level for tofogliflozin (range of $10 \mathrm{mg}$ to $40 \mathrm{mg}$ ) compared to placebo.

the results of metaregression, shown in Table 3, demonstrated that the amount of SUA change was not significantly correlated with dosage and weeks of treatment. However, SUA change was positively correlated with the duration of diabetes $\quad($ Coefficient $=1.581 \quad[0.148, \quad 3.014] ; \quad P=0.03)$ (Table 3). Figure S4 shows the scatter plots of metaregression by week, SGLT2i dosage, and duration of diabetes covariates. Figures S7, S10, and S13 show the 

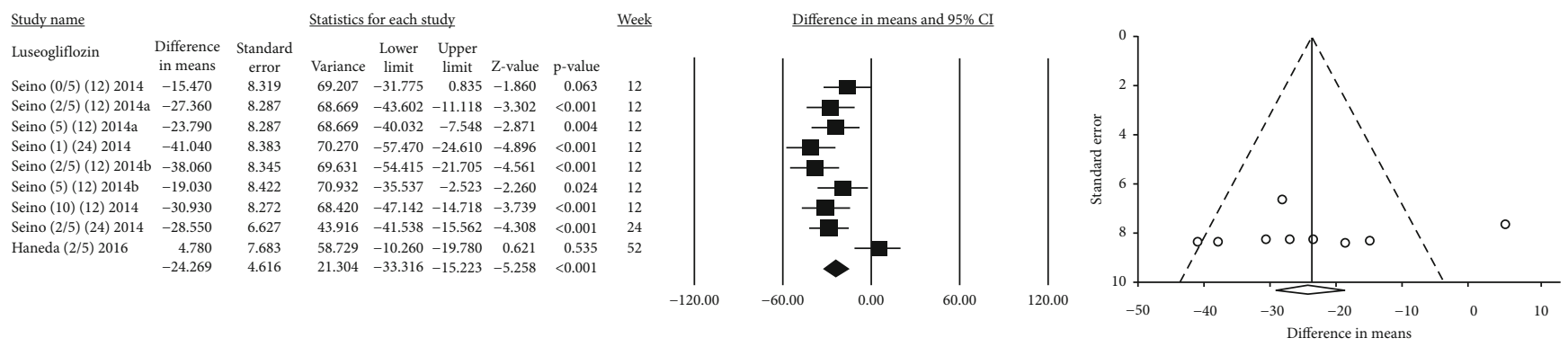

Figure 7: Mean difference and 95\% confidence intervals for changes in serum uric acid level for luseogliflozin (range of $0.5 \mathrm{mg}$ to $10 \mathrm{mg}$ ) compared to placebo.

TABLE 3: The results of metaregression analysis on the effects of SGLT2i on SUA reduction based on duration of diabetes, treatment duration, and SGLT2i dosage.

\begin{tabular}{|c|c|c|c|c|c|c|c|c|c|c|}
\hline & \multicolumn{3}{|c|}{ Canagliflozin } & \multicolumn{4}{|c|}{ Dapagliflozin } & \multicolumn{3}{|c|}{ Empagliflozin } \\
\hline & $100 \mathrm{mg}$ & $300 \mathrm{mg}$ & Total & $2.5 \mathrm{mg}$ & $5 \mathrm{mg}$ & $10 \mathrm{mg}$ & Total & $10 \mathrm{mg}$ & $25 \mathrm{mg}$ & Total \\
\hline \multicolumn{11}{|l|}{ Week } \\
\hline Coefficient & $\begin{array}{c}0.329 \\
{[-1.400} \\
2.059]\end{array}$ & $\begin{array}{c}-0.524 \\
{[-1.674} \\
0.625]\end{array}$ & $\begin{array}{c}0.026 \\
{[-0.751} \\
0.805]\end{array}$ & - & $\begin{array}{c}-0.030 \\
{[-0.587} \\
0.523]\end{array}$ & $\begin{array}{c}0.236 \\
{[-0.302} \\
0.776]\end{array}$ & $\begin{array}{c}0.124 \\
{[-0.255} \\
0.505]\end{array}$ & $\begin{array}{c}0.550 \\
{[0.068,} \\
1.03]\end{array}$ & $\begin{array}{c}0.634 \\
{[0.089,} \\
1.179]\end{array}$ & $\begin{array}{c}0.607 \\
{[0.282,} \\
0.931]\end{array}$ \\
\hline$P$-value & 0.709 & 0.371 & 0.946 & - & 0.913 & 0.389 & 0.520 & 0.025 & 0.022 & $<0.001$ \\
\hline \multicolumn{11}{|l|}{ Dosage } \\
\hline Coefficient & - & - & $\begin{array}{c}-0.013 \\
{[-0.046} \\
0.018]\end{array}$ & - & - & - & $\begin{array}{c}-0.606 \\
{[-2.055} \\
0.842]\end{array}$ & - & - & $\begin{array}{c}-0.278 \\
{[-0.788} \\
0.232]\end{array}$ \\
\hline$P$-value & - & - & 0.411 & - & - & - & 0.411 & - & - & 0.285 \\
\hline \multicolumn{11}{|c|}{ Duration of diabetes } \\
\hline Coefficient & $\begin{array}{c}2.061 \\
{[-0.359} \\
4.481]\end{array}$ & $\begin{array}{c}1.286 \\
{[-0.691} \\
3.264]\end{array}$ & $\begin{array}{c}1.581 \\
{[0.148,} \\
3.014]\end{array}$ & $\begin{array}{c}1.746 \\
{[0.503,} \\
2.990]\end{array}$ & $\begin{array}{c}2.109 \\
{[1.254,} \\
2.964]\end{array}$ & $\begin{array}{c}2.076 \\
{[0.889} \\
3.263]\end{array}$ & $\begin{array}{l}1.906 \\
{[1.218,} \\
2.594]\end{array}$ & - & - & - \\
\hline$P$-value & 0.095 & 0.202 & 0.03 & 0.005 & $<0.001$ & $<0.001$ & $<0.001$ & - & - & - \\
\hline
\end{tabular}

forest plot of HbAlc, FPG, and body weight changes, respectively.

3.1.2. Dapagliflozin. Eighteen clinical trials with a total of 41 comparisons examined the effect of dapagliflozin (range of $1 \mathrm{mg}$ to $50 \mathrm{mg}$ ) on SUA levels. The pooled effects of different doses of dapagliflozin on SUA, HbAlc, and FPG are reported in Table 2. MD of HbA1c, body weight, and FPG changes was lower in dapagliflozin studies than other types of SGLT2i. Furthermore, the results of random-effects meta-regression indicated that the amount of SUA change does not correlate with dosage or weeks of treatment, but SUA change was positively correlated with the duration of diabetes (Coefficient $=1.906 \quad[1.218,2.594] ; \quad P<0.001)$ (Table 3). Figure S5 shows the scatter plots of metaregression by week, dosage, and duration of diabetes covariates. Figures S8, S11, and S14 show the forest plot of HbA1c, FPG, and body weight changes, respectively. Moreover, one study was removed because it was conducted on prediabetic patients. The findings showed that dapagliflozin reduced SUA levels $(\mathrm{MD}=-62 \pm 47 \mu \mathrm{mol} / \mathrm{L})[45]$.
3.1.3. Empagliflozin. Seventeen trials assessed the effect of empagliflozin (range of $5 \mathrm{mg}$ to $100 \mathrm{mg}$ ) on SUA. Empagliflozin had the highest rate of SUA reduction $(\mathrm{MD}=-40.98$ ; CI [-47.63, -34.32]; $\left.I^{2}=84.9 \%\right)$. Empagliflozin effects on SUA, HbA1c, body weight, and FPG are shown in Table 2. Scatter plots of metaregression by the week of treatment and dosage covariates are shown in Figure S6. Figures S9, S12, and S15 show the forest plot of HbA1c, FPG, and body weight changes, respectively. We removed the Zanchi et al. study from the meta-analysis. They employed nondiabetic patients to measure the effect of empagliflozin $10 \mathrm{mg}$; the results also showed a reduction in SUA $(\mathrm{MD}=-97 \pm 36 \mu \mathrm{mol} / \mathrm{L})[70]$.

3.1.4. Other SGLT2i. The effects of other SGLT2is on SUA, HbAlc, body weight, and FPG are also reported in Table 2. Three studies assessed ipragliflozin (range of $12.5 \mathrm{mg}$ to $300 \mathrm{mg}$ ), two studies assessed tofogliflozin (range of $10 \mathrm{mg}$ to $40 \mathrm{mg}$ ), and four studies assessed luseogliflozin (range of $0.5 \mathrm{mg}$ to $10 \mathrm{mg}$ ) effects on SUA levels. Four studies were removed from the meta-analysis because they did not assess patients with T2DM. A recent study in 2020 
assessed the effects of 12 weeks of treatment with licogliflozin on 123 obese patients. MD of SUA change in different doses was between -65.1 and $-74.4 \mu \mathrm{mol} / \mathrm{L}$ [69]. Van Raalte et al. in 2019 assessed a 24-week treatment with sotagliflozin $200 \mathrm{mg}$ or $400 \mathrm{mg}$ on 955 type 1 diabetes patients compared with 479 patients in the placebo group; MD of SUA was calculated $-32.71 \pm 38.95 \mu \mathrm{mol} / \mathrm{L}$ [63].

3.2. Publication Bias. Regarding Egger's test, canagliflozin $100 \mathrm{mg}$, canagliflozin $300 \mathrm{mg}$, empagliflozin $10 \mathrm{mg}$, and empagliflozin $25 \mathrm{mg}$ had publication bias $(P-$ value $<0.05$ ). However, Begg's test did not show any publication bias, except for canagliflozin (total).

\section{Discussion}

The current meta-analysis of 55 placebo-controlled trials analyzed the data of 23,494 patients who received SGLT2is compared with 12,721 patients who received a placebo. The mean difference of SUA changes was about $-34.07 \mu \mathrm{mol} / \mathrm{L}$ (95\% CI [-37.00, -31.14], $I^{2}=78.8 \%$ ) among T2DM patients. Empagliflozin showed more potential in SUA reduction than other SGLT2is, while ipragliflozin had the least SUA changes.

There are six meta-analyses on this topic, two of which focused specifically on SUA change. Wu et al. assessed the impact of SGLT2i as an add-on treatment to insulin therapy compared to the control group in patients with T2DM, which received a placebo in addition to insulin. They calculated MD of SUA change $-26.16 \mu \mathrm{mol} / \mathrm{L}$ (95\% CI [-42.14, -10.17], $I^{2}$ $=80 \%)$ through assessment of 5 comparisons [78]. Yumo Zhao et al. specifically focused on SUA changes of 62 trials, which compared the effects of SGLT2is with placebo or active control or standard care. Overall MD of SUA changes was $-37.73 \mu \mathrm{mol} / \mathrm{L}$ (CI [-40.51, -34.95], $I^{2}=73.5 \%$ ) [79].

Dapagliflozin was studied more than the other SGLT2is. In accordance with our study, a previous meta-analysis on 4454 patients showed that dapagliflozin can significantly reduce SUA; the weighted mean difference (WMD) of SUA changes was about $-41.50 \mu \mathrm{mol} / \mathrm{L}$ (95\% CI [-47.22, -35.79]; $\left.I^{2}=50 \%\right)$, while it was about $-35.17 \mu \mathrm{mol} / \mathrm{L} \quad(95 \%$ CI $\left.[-39.68,-30.66], I^{2}=73.9 \%\right)$ in our study [80]. The effects of dapagliflozin on SUA were also assessed by Zhang et al. on 5302 patients, with results being similar to those of our study (WMD $-36.17 \mu \mathrm{mol} / \mathrm{L} ; 95 \%$ CI: $-40.99,-31.36 ; I^{2}=64$ \%) [81]. Both studies compared dapagliflozin with a placebo.

In agreement with us, Xin et al. assessed SUA changes obtained with 5 types of SGLT2i compared with placebo or control or standard care. All SGLT2is significantly decreased SUA levels compared with placebo; canagliflozin WMD $37.02 \mu \mathrm{mol} / \mathrm{L} \quad(95 \% \quad$ CI $[-38.41,-35.63])$, dapagliflozin WMD $-38.05 \mu \mathrm{mol} / \mathrm{L}$ (95\% CI [-44.47, -31.62]), empagliflozin WMD $-42.07 \mu \mathrm{mol} / \mathrm{L}(95 \%$ CI $[-46.27,-37.86])$, tofogliflozin WMD $-18.97 \mu \mathrm{mol} / \mathrm{L}$ (95\% CI $[-28.79,-9.16])$, and ipragliflozin WMD -19.75 $\mu \mathrm{mol} / \mathrm{L}$ (95\% CI [-28.17, -11.34]) [76]. Furthermore, Yumo Zhao et al. performed a metaregression and concluded that only the effect of dapagliflozin depended on the administration dosage. In addition, the metaregression of Yumo Zhao et al. showed that the reduc- tion of SUA could be persistent with long-term, 104-week administration of SGLT2is [79]. Conversely, our study showed no relationship between SUA reduction and duration (except for empagliflozin) and dosage of SGLT2i. However, our data showed that SUA was reduced more in the canagliflozin and dapagliflozin groups, with a more pronounced reduction observed in patients with a longer duration of diabetes. Perhaps, longer duration of diabetes may alter the expression of SGLT2, glucose transporter 9 (GLUT9), or related unknown pathways in the kidney, thus favouring uric acid excretion. Di Zhao et al. specifically reviewed the effect of empagliflozin on some cardiometabolic risk factors [75]. In accordance with our review, they showed that empagliflozin could significantly reduce SUA level, HbAlc, and FPG. However, there are some differences: the mean change of HbAlc and FPG, unlike SUA, was higher in their study. The differences may be due to the mean treatment period, the number of patients, and different analysis tools.

Increased SUA causes inflammation in adipocytes as well as endothelial dysfunction, which reduces nitric oxide bioavailability and leads to insulin resistance. Moreover, uric acid impairs glucose uptake in skeletal muscle, which reduces insulin-stimulated glucose uptake [82]. Insulin resistance leads to hyperinsulinemia, which elevates SUA through lowering renal uric acid excretion [83, 84].

SGLT2is could significantly decrease SUA through several mechanisms. GLUT9 protein is expressed in two subtypes, namely, GLUT9a and GLUT9b, localized in the apical and basolateral membrane of the proximal tubule, respectively. GLUT9 subtypes regulate uric acid transportation and concentration [85]. Chino et al. revealed that the urinary excretion rate of uric acid strongly correlated with the urinary glucose excretion, demonstrating the relation between SUA and glycosuria [86]. Raised glucose concentration resulting from SGLT2i administration could also disturb the reabsorption of uric acid in the proximal tubule through GLUT9b [87]. After removing the studies that were conducted on CKD patients, the SUA reduction was increased, which is consistent with the proposed model for uricosuric effects of SGLT2i by Chino et al. [86]. Hence, SGLT2i induce more pronounced glycosuria in the presence of higher eGFR values. Moreover, part of the SUA reduction can be explained by the body weight loss induced by SGLT2is. Previous studies showed a strong positive correlation between body mass index and SUA levels [88-91]. Body weight loss is recommended for the management of gout $[92,93]$. A possible explanation is that insulin resistance increases the reabsorption of organic anions like urate [94].

4.1. Limitations and Strengths. Our study has some limitations. First, due to the paucity of available studies, we could not perform a meta-analysis for ertugliflozin and sotagliflozin. Second, some studies did not report the standard deviation or related data to calculate it. Third, trials with CKD patients, whose plasma UA level may be increased because of disease deterioration, could interfere with the results. Fourth, some studies had some dropouts, but they reported 
baseline data of all patients. Fifth, the baseline SUA level and follow-up period were different across the studies. Sixth, some of the administered doses of SGLT2i were not within the approved dose range for the T2DM treatment. Finally, the heterogeneity of SUA data was moderate or high except for canagliflozin $(300 \mathrm{mg})$ and tofogliflozin. The comparison with active control groups and paucity of available studies were the other limitations of previous meta-analyses.

4.2. Conclusion. All SGLT2i analyzed in the meta-analysis can reduce SUA in patients with T2DM $(\mathrm{MD}=-34.076$; CI $[-37.006,-31.146])$. The ability to reduce SUA is one of the advantages of SGLT2is over other antidiabetic medications, particularly in patients with T2DM and comorbid hyperuricemia. Moreover, the urate-lowering properties exerted by SGLT2i may partly explain their wellestablished renoprotective and cardioprotective actions. More placebo-controlled studies are warranted for luseogliflozin, licogliflozin, sotagliflozin, and ertugliflozin to clarify their effects on SUA.

\section{Data Availability}

There is no raw data associated with this article.

\section{Conflicts of Interest}

The authors declare that they have no conflicts of interest.

\section{Supplementary Materials}

Table S1: search strategy. Figure S1: meta-analysis of all canagliflozin studies to determine the drug efficacy in serum uric acid reduction. Figure S2: meta-analysis of all dapagliflozin studies to determine the drug efficacy in serum uric acid reduction. Figure S3: meta-analysis of all empagliflozin studies to determine the drug efficacy in serum uric acid reduction. Figure S4: scatterplots of metaregression on canagliflozin variables (weeks of treatment, drug dosage, and duration of diabetes). Figure S5: scatterplots of metaregression on dapagliflozin variables (weeks of treatment, drug dosage, and duration of diabetes). Figure S6: scatterplots of metaregression on empagliflozin variables (weeks of treatment, drug dosage, and duration of diabetes). Figure S7: meta-analysis of all canagliflozin studies to determine the drug effects on HbAlc. Figure S8: meta-analysis of all dapagliflozin studies to determine the drug effects on HbAlc. Figure S9: meta-analysis of all empagliflozin studies to determine the drug effects on HbAlc. Figure S10: meta-analysis of all canagliflozin studies to determine the drug effects on FPG. Figure S11: meta-analysis of all dapagliflozin studies to determine the drug effects on FPG. Figure S12: metaanalysis of all empagliflozin studies to determine the drug effects on FPG. Figure S13: meta-analysis of all canagliflozin studies to determine the drug effects on body weight. Figure S14: meta-analysis of all dapagliflozin studies to determine the drug effects on body weight. Figure S15: meta-analysis of all empagliflozin studies to determine the drug effects on body weight. (Supplementary Materials)

\section{References}

[1] V. Tat and C. P. Forest, "The role of SGLT2 inhibitors in managing type 2 diabetes," JAAPA, vol. 31, no. 6, pp. 35-40, 2018.

[2] H. Yaribeygi, A. E. Butler, S. L. Atkin, N. Katsiki, and A. Sahebkar, "Sodium-glucose cotransporter 2 inhibitors and inflammation in chronic kidney disease: possible molecular pathways," Journal of cellular physiology, vol. 234, no. 1, pp. 223-230, 2019.

[3] H. Yaribeygi, L. E. Simental-Mendía, M. Banach, S. Bo, and A. Sahebkar, "The major molecular mechanisms mediating the renoprotective effects of SGLT2 inhibitors: an update," Biomedicine \& Pharmacotherapy, vol. 120, p. 109526, 2019.

[4] T. D. Filippatos, A. Liontos, I. Papakitsou, and M. S. Elisaf, "SGLT2 inhibitors and cardioprotection: a matter of debate and multiple hypotheses," Postgraduate Medicine, vol. 131, no. 2, pp. 82-88, 2019.

[5] M. Ashrafizadeh, H. Yaribeygi, S. L. Atkin, and A. Sahebkar, "Effects of newly introduced antidiabetic drugs on autophagy," Diabetes \& Metabolic Syndrome: Clinical Research \& Reviews, vol. 13, no. 4, pp. 2445-2449, 2019.

[6] Z. Liu, X. Ma, I. Ilyas et al., "Impact of sodium glucose cotransporter 2 (SGLT2) inhibitors on atherosclerosis: from pharmacology to pre-clinical and clinical therapeutics," Theranostics, vol. 11, no. 9, pp. 4502-4515, 2021.

[7] H. Yaribeygi, N. Katsiki, A. E. Butler, and A. Sahebkar, "Effects of antidiabetic drugs on NLRP3 inflammasome activity, with a focus on diabetic kidneys," Drug Discovery Today, vol. 24, no. 1, pp. 256-262, 2019.

[8] G. Ranjbar, D. P. Mikhailidis, and A. Sahebkar, "Effects of newer antidiabetic drugs on nonalcoholic fatty liver and steatohepatitis: think out of the box!," Metabolism: Clinical and Experimental, vol. 101, 2019.

[9] H. Yaribeygi, M. Ashrafizadeh, N. C. Henney, T. Sathyapalan, T. Jamialahmadi, and A. Sahebkar, "Neuromodulatory effects of anti-diabetes medications: A mechanistic review," Pharmacological Research, vol. 152, p. 104611, 2020.

[10] H. Yaribeygi, S. L. Atkin, A. E. Butler, and A. Sahebkar, "Sodium-glucose cotransporter inhibitors and oxidative stress: an update," Journal of Cellular Physiology, vol. 234, no. 4, pp. 3231-3237, 2019.

[11] H. Yaribeygi, S. L. Atkin, T. Jamialahmadi, and A. Sahebkar, "A review on the effects of new anti-diabetic drugs on platelet function," Endocrine, Metabolic and Immune Disorders-Drug Targets, vol. 20, no. 3, pp. 328-334, 2020.

[12] H. Yaribeygi, S. L. Atkin, and A. Sahebkar, "Mechanistic effects of SGLT2 inhibition on blood pressure in diabetes," Diabetes and Metabolic Syndrome: Clinical Research and Reviews, vol. 13, no. 2, pp. 1679-1683, 2019.

[13] E. Araki, Y. Onishi, M. Asano, H. Kim, and T. Yajima, "Efficacy and safety of dapagliflozin over 1 year as add-on to insulin therapy in Japanese patients with type 2 diabetes: the DAISY (Dapagliflozin added to patients under InSulin therapY) trial," Diabetes, Obesity and Metabolism, vol. 19, no. 4, pp. 562-570, 2017.

[14] C. Bailey, N. Iqbal, C. Tjoen, and J. List, "Dapagliflozin monotherapy in drug-naïve patients with diabetes: a randomizedcontrolled trial of low-dose range," Diabetes, Obesity and Metabolism, vol. 14, no. 10, pp. 951-959, 2012.

[15] C. Bailey, E. Morales Villegas, V. Woo, W. Tang, A. Ptaszynska, and J. List, "Efficacy and safety of dapagliflozin monotherapy in people with type 2 diabetes: a randomized 
double-blind placebo-controlled 102-week trial," Diabetic Medicine, vol. 32, no. 4, pp. 531-541, 2015.

[16] C. J. Bailey, J. L. Gross, A. Pieters, A. Bastien, and J. F. List, "Effect of dapagliflozin in patients with type 2 diabetes who have inadequate glycaemic control with metformin: a randomised, double-blind, placebo-controlled trial," The Lancet, vol. 375, no. 9733, pp. 2223-2233, 2010.

[17] B. Bode, K. Stenlöf, S. Harris et al., "Long-term efficacy and safety of canagliflozin over 104 weeks in patients aged 55-80 years with type 2 diabetes," Diabetes, Obesity and Metabolism, vol. 17, no. 3, pp. 294-303, 2015.

[18] B. Bode, K. Stenlöf, D. Sullivan, A. Fung, and K. Usiskin, "Efficacy and safety of canagliflozin treatment in older subjects with type 2 diabetes mellitus: a randomized trial," Hospital Practice, vol. 41, no. 2, pp. 72-84, 2013.

[19] J. Bolinder, Ö. Ljunggren, J. Kullberg et al., "Effects of dapagliflozin on body weight, total fat mass, and regional adipose tissue distribution in patients with type 2 diabetes mellitus with inadequate glycemic control on metformin," The Journal of Clinical Endocrinology \& Metabolism, vol. 97, no. 3, pp. 1020-1031, 2012.

[20] M. J. Budoff, T. M. Davis, A. G. Palmer et al., "Efficacy and safety of ertugliflozin in patients with type 2 diabetes inadequately controlled by metformin and sulfonylurea: a substudy of VERTIS CV," Diabetes Therapy, vol. 12, no. 5, pp. 1279-1297, 2021.

[21] E. Ferrannini, S. J. Ramos, A. Salsali, W. Tang, and J. F. List, "Dapagliflozin monotherapy in type 2 diabetic patients with inadequate glycemic control by diet and exercise: a randomized, double-blind, placebo-controlled, phase 3 trial," Diabetes Care, vol. 33, no. 10, pp. 22172224, 2010.

[22] P. Fioretto, S. Del Prato, J. B. Buse et al., "Efficacy and safety of dapagliflozin in patients with type 2 diabetes and moderate renal impairment (chronic kidney disease stage 3A): the DERIVE study," Diabetes, Obesity and Metabolism, vol. 20, no. 11, pp. 2532-2540, 2018.

[23] M. Haneda, Y. Seino, N. Inagaki et al., "Influence of renal function on the 52-week efficacy and safety of the sodium glucose cotransporter 2 inhibitor luseogliflozin in Japanese patients with type 2 diabetes mellitus," Clinical therapeutics, vol. 38 , no. 1, pp. 66-88.e20, 2016.

[24] H. U. Häring, L. Merker, E. Seewaldt-Becker et al., "Empagliflozin as add-on to metformin plus sulfonylurea in patients with type 2 diabetes: a 24-week, randomized, double-blind, placebo-controlled trial," Diabetes Care, vol. 36, no. 11, pp. 3396-3404, 2013.

[25] T. Heise, E. Seewaldt-Becker, S. Macha et al., "Safety, tolerability, pharmacokinetics and pharmacodynamics following 4 weeks' treatment with empagliflozin once daily in patients with type 2 diabetes," Diabetes, Obesity and Metabolism, vol. 15, no. 7, pp. 613-621, 2013.

[26] R. Henry, A. Murray, M. Marmolejo, D. Hennicken, A. Ptaszynska, and J. List, "Dapagliflozin, metformin XR, or both: initial pharmacotherapy for type 2 diabetes, a randomised controlled trial," International Journal of Clinical Practice, vol. 66, no. 5, pp. 446-456, 2012.

[27] S. A. Jabbour, E. Hardy, J. Sugg, S. Parikh, and Group S, "Dapagliflozin is effective as add-on therapy to sitagliptin with or without metformin: a 24-week, multicenter, randomized, double-blind, placebo-controlled study," Diabetes Care, vol. 37, no. 3, pp. 740-750, 2014.
[28] L. Ji, P. Han, Y. Liu et al., "Canagliflozin in Asian patients with type 2 diabetes on metformin alone or metformin in combination with sulphonylurea," Diabetes, Obesity and Metabolism, vol. 17, no. 1, pp. 23-31, 2015.

[29] L. Ji, J. Ma, H. Li et al., "Dapagliflozin as monotherapy in drug-naive Asian patients with type 2 diabetes mellitus: a randomized, blinded, prospective phase III study," Clinical therapeutics, vol. 36, no. 1, pp. 84-100.e9, 2014.

[30] T. Kadowaki, M. Haneda, N. Inagaki et al., "Empagliflozin monotherapy in Japanese patients with type 2 diabetes mellitus: a randomized, 12-week, double-blind, placebo-controlled, phase II trial," Advances in Therapy, vol. 31, no. 6, pp. 621638, 2014.

[31] S. Kahl, S. Gancheva, K. Straßburger et al., "Empagliflozin effectively lowers liver fat content in well-controlled type 2 diabetes: a randomized, double-blind, phase 4, placebo-controlled trial," Diabetes Care, vol. 43, no. 2, pp. 298-305, 2020.

[32] K. Kaku, H. Watada, Y. Iwamoto et al., "Efficacy and safety of monotherapy with the novel sodium/glucose cotransporter-2 inhibitor tofogliflozin in Japanese patients with type 2 diabetes mellitus: a combined phase 2 and 3 randomized, placebo-controlled, double-blind, parallel-group comparative study," Cardiovascular Diabetology, vol. 13, no. 1, pp. 65-115, 2014.

[33] K. Kario, K. Okada, M. Kato et al., "Twenty-four-hour blood pressure-lowering effect of a sodium-glucose cotransporter 2 inhibitor in patients with diabetes and uncontrolled nocturnal Hypertension," Circulation, vol. 139, no. 18, pp. 2089-2097, 2019.

[34] A. Kashiwagi, K. Kazuta, Y. Takinami, S. Yoshida, A. Utsuno, and I. Nagase, "Ipragliflozin improves glycemic control in Japanese patients with type 2 diabetes mellitus: the BRIGHTEN study," Diabetology International, vol. 6, no. 1, pp. 8-18, 2015.

[35] A. Kashiwagi, K. Kazuta, S. Yoshida, and I. Nagase, "Randomized, placebo-controlled, double-blind glycemic control trial of novel sodium-dependent glucose cotransporter 2 inhibitor ipragliflozin in Japanese patients with type 2 diabetes mellitus," Journal of Diabetes Investigation, vol. 5, no. 4, pp. 382391, 2014.

[36] D. E. Kohan, P. Fioretto, W. Tang, and J. F. List, "Long-term study of patients with type 2 diabetes and moderate renal impairment shows that dapagliflozin reduces weight and blood pressure but does not improve glycemic control," Kidney International, vol. 85, no. 4, pp. 962-971, 2014.

[37] C. S. Kovacs, V. Seshiah, L. Merker et al., "Empagliflozin as add-on therapy to pioglitazone with or without metformin in patients with type 2 diabetes mellitus," Clinical Therapeutics, vol. 37, no. 8, pp. 1773-1788.e1, 2015.

[38] J. Li, S. V. Badve, Z. Zhou et al., "The effects of canagliflozin on uric acid and gout in patients with type 2 diabetes in the CANVAS Program," Diabetes, vol. 68, Supplement 1, pp. 12161217, 2019.

[39] J. F. List, V. Woo, E. Morales, W. Tang, and F. T. Fiedorek, "Sodium-glucose cotransport inhibition with dapagliflozin in type 2 diabetes," Diabetes Care, vol. 32, no. 4, pp. 650-657, 2009.

[40] L.-L. Ma, Y.-Y. Wang, Z.-H. Yang, D. Huang, H. Weng, and X.-T. Zeng, "Methodological quality (risk of bias) assessment tools for primary and secondary medical studies: what are they and which is better?," Military Medical Research, vol. 7, no. 1, pp. 1-11, 2020.

[41] N. A. Mordi, I. R. Mordi, J. S. Singh, R. J. McCrimmon, A. D. Struthers, and C. C. Lang, "Renal and cardiovascular effects of 
SGLT2 inhibition in combination with loop diuretics in patients with type 2 diabetes and chronic heart failure: the RECEDE-CHF trial," Circulation, vol. 142, no. 18, pp. 17131724, 2020.

[42] R. Nishimura, Y. Tanaka, K. Koiwai et al., "Effect of empagliflozin monotherapy on postprandial glucose and 24-hour glucose variability in Japanese patients with type 2 diabetes mellitus: a randomized, double-blind, placebo-controlled, 4week study," Cardiovascular Diabetology, vol. 14, no. 1, pp. 1-13, 2015.

[43] C. Pollock, B. Stefánsson, D. Reyner et al., “Albuminuria-lowering effect of dapagliflozin alone and in combination with saxagliptin and effect of dapagliflozin and saxagliptin on glycaemic control in patients with type 2 diabetes and chronic kidney disease (DELIGHT): a randomised, double-blind, placebo-controlled trial," The Lancet Diabetes \& Endocrinology, vol. 7, no. 6, pp. 429-441, 2019.

[44] R. Qiu, G. Capuano, and G. Meininger, "Efficacy and safety of twice-daily treatment with canagliflozin, a sodium glucose cotransporter 2 inhibitor, added on to metformin monotherapy in patients with type 2 diabetes mellitus," Journal of Clinical \& Translational Endocrinology, vol. 1, no. 2, pp. 54-60, 2014.

[45] A. M. Ramírez-Rodríguez, M. González-Ortiz, and E. Martínez-Abundis, "Effect of dapagliflozin on insulin secretion and insulin sensitivity in patients with prediabetes," Experimental and Clinical Endocrinology \& Diabetes, vol. 128, no. 8, pp. 506-511, 2020.

[46] H. Rodbard, J. Seufert, N. Aggarwal et al., "Efficacy and safety of titrated canagliflozin in patients with type 2 diabetes mellitus inadequately controlled on metformin and sitagliptin," Diabetes, Obesity and Metabolism, vol. 18, no. 8, pp. 812819, 2016.

[47] M. Roden, L. Merker, A. V. Christiansen et al., "Safety, tolerability and effects on cardiometabolic risk factors of empagliflozin monotherapy in drug-naïve patients with type 2 diabetes: a double-blind extension of a phase III randomized controlled trial," Cardiovascular Diabetology, vol. 14, no. 1, pp. 1-11, 2015.

[48] J. Rosenstock, N. Aggarwal, D. Polidori et al., "Dose-ranging effects of canagliflozin, a sodium-glucose cotransporter 2 inhibitor, as add-on to metformin in subjects with type 2 diabetes," Diabetes Care, vol. 35, no. 6, pp. 1232-1238, 2012.

[49] J. Rosenstock, A. Jelaska, G. Frappin et al., "Improved glucose control with weight loss, lower insulin doses, and no increased hypoglycemia with empagliflozin added to titrated multiple daily injections of insulin in obese inadequately controlled type 2 diabetes," Diabetes Care, vol. 37, no. 7, pp. 1815-1823, 2014.

[50] J. Rosenstock, A. Jelaska, C. Zeller et al., "Impact of empagliflozin added on to basal insulin in type 2 diabetes inadequately controlled on basal insulin: a 78-week randomized, doubleblind, placebo-controlled trial," Diabetes, Obesity and Metabolism, vol. 17, no. 10, pp. 936-948, 2015.

[51] J. Rosenstock, M. Vico, L. Wei, A. Salsali, and J. F. List, "Effects of dapagliflozin, an SGLT2 inhibitor, on HbA1c, body weight, and hypoglycemia risk in patients with type 2 diabetes inadequately controlled on pioglitazone monotherapy," Diabetes Care, vol. 35, no. 7, pp. 1473-1478, 2012.

[52] S. Ross, C. Thamer, J. Cescutti, T. Meinicke, H. Woerle, and U. Broedl, "Efficacy and safety of empagliflozin twice daily versus once daily in patients with type 2 diabetes inadequately controlled on metformin: a 16-week, randomized, placebo- controlled trial," Diabetes, Obesity and Metabolism, vol. 17, no. 7, pp. 699-702, 2015.

[53] P. M. Schumm-Draeger, L. Burgess, L. Korányi, V. Hruba, J. Hamer-Maansson, and T. de Bruin, "Twice-daily dapagliflozin co-administered with metformin in type 2 diabetes: a 16week randomized, placebo-controlled clinical trial," Diabetes, Obesity and Metabolism, vol. 17, no. 1, pp. 42-51, 2015.

[54] Y. Seino, T. Sasaki, A. Fukatsu, S. Sakai, and Y. Samukawa, "Efficacy and safety of luseogliflozin monotherapy in Japanese patients with type 2 diabetes mellitus: a 12-week, randomized, placebo-controlled, phase II study," Current Medical Research and Opinion, vol. 30, no. 7, pp. 1219-1230, 2014.

[55] Y. Seino, T. Sasaki, A. Fukatsu, M. Ubukata, S. Sakai, and Y. Samukawa, "Dose-finding study of luseogliflozin in Japanese patients with type 2 diabetes mellitus: a 12-week, randomized, double-blind, placebo-controlled, phase II study," Current Medical Research and Opinion, vol. 30, no. 7, pp. 1231-1244, 2014.

[56] S. Sha, D. Polidori, T. Heise et al., "Effect of the sodium glucose co-transporter 2 inhibitor canagliflozin on plasma volume in patients with type 2 diabetes mellitus," Diabetes, Obesity and Metabolism, vol. 16, no. 11, pp. 1087-1095, 2014.

[57] W. Shimizu, Y. Kubota, Y. Hoshika et al., "Effects of empagliflozin versus placebo on cardiac sympathetic activity in acute myocardial infarction patients with type 2 diabetes mellitus: the EMBODY trial," Cardiovascular Diabetology, vol. 19, no. 1, pp. 1-12, 2020.

[58] E. Søfteland, J. J. Meier, B. Vangen, R. Toorawa, M. Maldonado-Lutomirsky, and U. C. Broedl, "Empagliflozin as add-on therapy in patients with type 2 diabetes inadequately controlled with linagliptin and metformin: a 24-week randomized, double-blind, parallel-group trial," Diabetes Care, vol. 40, no. 2, pp. 201-209, 2017.

[59] K. Stenlöf, W. Cefalu, K. A. Kim et al., "Efficacy and safety of canagliflozin monotherapy in subjects with type 2 diabetes mellitus inadequately controlled with diet and exercise," Diabetes, Obesity and Metabolism, vol. 15, no. 4, pp. 372-382, 2013.

[60] K. Strojek, K. Yoon, V. Hruba, M. Elze, A. Langkilde, and S. Parikh, "Effect of dapagliflozin in patients with type 2 diabetes who have inadequate glycaemic control with glimepiride: a randomized, 24-week, double-blind, placebo-controlled trial," Diabetes, Obesity and Metabolism, vol. 13, no. 10, pp. 928-938, 2011.

[61] Y. Terauchi, M. Tamura, M. Senda, R. Gunji, and K. Kaku, "Efficacy and safety of tofogliflozin in Japanese patients with type 2 diabetes mellitus with inadequate glycaemic control on insulin therapy (J-STEP/INS): results of a 16-week randomized, double-blind, placebo-controlled multicentre trial," Diabetes, Obesity and Metabolism, vol. 19, no. 10, pp. 13971407, 2017.

[62] I. Tikkanen, K. Narko, C. Zeller et al., "Empagliflozin reduces blood pressure in patients with type 2 diabetes and hypertension," Diabetes Care, vol. 38, no. 3, pp. 420-428, 2015.

[63] D. H. van Raalte, P. Bjornstad, F. Persson et al., "The impact of sotagliflozin on renal function, albuminuria, blood pressure, and hematocrit in adults with type 1 diabetes," Diabetes Care, vol. 42, no. 10, pp. 1921-1929, 2019.

[64] M. A. Weber, T. A. Mansfield, F. Alessi, N. Iqbal, S. Parikh, and A. Ptaszynska, "Effects of dapagliflozin on blood pressure in hypertensive diabetic patients on renin-angiotensin system blockade," Blood Pressure, vol. 25, no. 2, pp. 93-103, 2016. 
[65] M. A. Weber, T. A. Mansfield, V. A. Cain, N. Iqbal, S. Parikh, and A. Ptaszynska, "Blood pressure and glycaemic effects of dapagliflozin versus placebo in patients with type 2 diabetes on combination antihypertensive therapy: a randomised, double-blind, placebo-controlled, phase 3 study," The Lancet Diabetes \& Endocrinology, vol. 4, no. 3, pp. 211-220, 2016.

[66] J. Wilding, E. Ferrannini, V. Fonseca, W. Wilpshaar, P. Dhanjal, and A. Houzer, "Efficacy and safety of ipragliflozin in patients with type 2 diabetes inadequately controlled on metformin: a dose-finding study," Diabetes, Obesity and Metabolism, vol. 15, no. 5, pp. 403-409, 2013.

[67] J. P. Wilding, V. Woo, N. G. Soler et al., "Long-term efficacy of dapagliflozin in patients with type 2 diabetes mellitus receiving high doses of insulin: a randomized trial," Annals of Internal Medicine, vol. 156, no. 6, pp. 405-415, 2012.

[68] J. F. Yale, G. Bakris, B. Cariou et al., "Efficacy and safety of canagliflozin over 52 weeks in patients with type 2 diabetes mellitus and chronic kidney disease," Diabetes, Obesity and Metabolism, vol. 16, no. 10, pp. 1016-1027, 2014.

[69] K. Yokote, M. Sano, I. Tsumiyama, and D. Keefe, "Dosedependent reduction in body weight with LIK066 (licogliflozin) treatment in Japanese patients with obesity," Diabetes, Obesity and Metabolism, vol. 22, no. 7, pp. 1102-1110, 2020.

[70] A. Zanchi, M. Burnier, M. E. Muller et al., "Acute and chronic effects of SGLT2 inhibitor empagliflozin on renal oxygenation and blood pressure control in nondiabetic normotensive subjects: a randomized, placebo-controlled trial," Journal of the American Heart Association, vol. 9, no. 13, article e016173, 2020.

[71] B. Zinman, C. Wanner, J. M. Lachin et al., "Empagliflozin, cardiovascular outcomes, and mortality in type 2 diabetes," New England Journal of Medicine, vol. 373, no. 22, pp. 2117-2128, 2015.

[72] A. H. Barnett, A. Mithal, J. Manassie et al., "Efficacy and safety of empagliflozin added to existing antidiabetes treatment in patients with type 2 diabetes and chronic kidney disease: a randomised, double-blind, placebo-controlled trial," The Lancet Diabetes \& Endocrinology, vol. 2, no. 5, pp. 369-384, 2014.

[73] L. Gaita, R. Timar, N. Lupascu et al., "The impact of hyperuricemia on cardiometabolic risk factors in patients with diabetes mellitus: a cross-sectional study," Diabetes, Metabolic Syndrome and Obesity: Targets and Therapy, vol. 12, pp. 20032010, 2019.

[74] V. Bartáková, K. Kuricová, L. Pácal et al., "Hyperuricemia contributes to the faster progression of diabetic kidney disease in type 2 diabetes mellitus," Journal of Diabetes and its Complications, vol. 30, no. 7, pp. 1300-1307, 2016.

[75] D. Zhao, H. Liu, and P. Dong, "Empagliflozin reduces blood pressure and uric acid in patients with type 2 diabetes mellitus: a systematic review and meta-analysis," Journal of Human Hypertension, vol. 33, no. 4, pp. 327-339, 2019.

[76] Y. Xin, Y. Guo, Y. Li, Y. Ma, L. Li, and H. Jiang, "Effects of sodium glucose cotransporter-2 inhibitors on serum uric acid in type 2 diabetes mellitus: a systematic review with an indirect comparison meta-analysis," Saudi Journal of Biological Sciences, vol. 26, no. 2, pp. 421-426, 2019.

[77] D. Moher, L. Shamseer, M. Clarke et al., "Preferred reporting items for systematic review and meta-analysis protocols (PRISMA-P) 2015 statement," Systematic Reviews, vol. 4, no. 1, pp. 1-9, 2015.

[78] B. Wu, H. Zheng, J. Gu et al., "Effects of sodium-glucose cotransporter 2 inhibitors in addition to insulin therapy on cardiovascular risk factors in type 2 diabetes patients: a meta-analysis of randomized controlled trials," Journal of Diabetes Investigation, vol. 10, no. 2, pp. 446-457, 2019.

[79] Y. Zhao, L. Xu, D. Tian et al., "Effects of sodium-glucose co-transporter 2 (SGLT2) inhibitors on serum uric acid level:Ameta-analysis of randomized controlled trials," Diabetes, Obesity and Metabolism, vol. 20, no. 2, pp. 458-462, 2018.

[80] G. Musso, R. Gambino, M. Cassader, and G. Pagano, "A novel approach to control hyperglycemia in type 2 diabetes: sodium glucose co-transport (SGLT) inhibitors. Systematic review and meta-analysis of randomized trials," Annals of Medicine, vol. 44, no. 4, pp. 375-393, 2012.

[81] M. Zhang, L. Zhang, B. Wu, H. Song, Z. An, and S. Li, "Dapagliflozin treatment for type 2 diabetes: a systematic review and meta-analysis of randomized controlled trials," Diabetes/ metabolism research and reviews, vol. 30, no. 3, pp. 204-221, 2014.

[82] A. F. Cicero, M. Rosticci, M. Bove et al., "Serum uric acid change and modification of blood pressure and fasting plasma glucose in an overall healthy population sample: data from the Brisighella heart study," Annals of Medicine, vol. 49, no. 4, pp. 275-282, 2017.

[83] T. Yamada, M. Fukatsu, S. Suzuki, T. Wada, and T. Joh, "Elevated serum uric acid predicts impaired fasting glucose and type 2 diabetes only among Japanese women undergoing health checkups," Diabetes \& Metabolism, vol. 37, no. 3, pp. 252-258, 2011.

[84] E. Muscelli, A. Natali, S. Bianchi et al., "Effect of insulin on renal sodium and uric acid handling in essential hypertension," American Journal of Hypertension, vol. 9, no. 8, pp. 746-752, 1996.

[85] B. Lüscher, D. Surbek, B. Clemençon et al., "Different pharmacological properties of GLUT9a and GLUT9b: potential implications in preeclampsia," Cellular Physiology and Biochemistry, vol. 53, no. 3, pp. 508-517, 2019.

[86] Y. Chino, Y. Samukawa, S. Sakai et al., "SGLT2 inhibitor lowers serum uric acid through alteration of uric acid transport activity in renal tubule by increased glycosuria," Biopharmaceutics \& Drug Disposition, vol. 35, no. 7, pp. 391-404, 2014.

[87] T. Takata and H. Isomoto, "Pleiotropic effects of sodiumglucose cotransporter-2 inhibitors: renoprotective mechanisms beyond glycemic control," International Journal of Molecular Sciences, vol. 22, no. 9, p. 4374, 2021.

[88] N. F. Chu, D. J. Wang, S. H. Liou, and S. M. Shieh, "Relationship between hyperuricemia and other cardiovascular disease risk factors among adult males in Taiwan," European Journal of Epidemiology, vol. 16, no. 1, pp. 13-17, 2000.

[89] H. Wang, L. Wang, R. Xie et al., "Association of serum uric acid with body mass index: a cross-sectional study from Jiangsu Province, China," Iranian Journal of Public Health, vol. 43, no. 11, pp. 1503-1509, 2014.

[90] S. M. Nielsen, E. M. Bartels, M. Henriksen et al., "Weight loss for overweight and obese individuals with gout: a systematic review of longitudinal studies," Annals of the Rheumatic Diseases, vol. 76, no. 11, pp. 1870-1882, 2017.

[91] J. Zhou, Y. Wang, F. Lian et al., "Physical exercises and weight loss in obese patients help to improve uric acid," Oncotarget, vol. 8, no. 55, pp. 94893-94899, 2017. 
[92] P. Richette, M. Doherty, E. Pascual et al., "2016 updated EULAR evidence-based recommendations for the management of gout," Annals of the Rheumatic Diseases, vol. 76, no. 1, pp. 29-42, 2017.

[93] M. Manara, A. Bortoluzzi, M. Favero et al., "Italian Society of Rheumatology recommendations for the management of gout," Reumatismo, vol. 65, no. 1, pp. 4-21, 2013.

[94] H. K. Choi, D. B. Mount, and A. M. Reginato, "Pathogenesis of gout," Annals of Internal Medicine, vol. 143, no. 7, pp. 499516, 2005.

[95] M. Roden, J. Weng, J. Eilbracht et al., "Empagliflozin monotherapy with sitagliptin as an active comparator in patients with type 2 diabetes: a randomised, double-blind, placebocontrolled, phase 3 trial," The Lancet Diabetes \& Endocrinology, vol. 1, no. 3, pp. 208-219, 2013. 